\title{
Fracture Failure Analysis of Fuel Pump Transmission Shaft of Dual-Fuel Engine
}

\author{
Pei-hong Chen, Jun-jie Zou and Li Fan \\ Marine Engineering Department, Nantong Shipping College, 226010, Nantong Jiangsu, China
}

\begin{abstract}
NTS6Z $\mathrm{L}$ Cz-129 dual-fuel turbocharged and intercooled engine durability test at $1000 \mathrm{~h}$, fuel pump shaft fractured. Fracture analysis, chemical analysis, microstructure examination and finite element stress analysis were carried out on the fractured shaft. The analysis result showed that the shaft fracture cause is forging fold. By improving the forging process, the forging fold was solved, and the durability test can be carried out smoothly.
\end{abstract}

\section{Introduction}

High pressure pump is driven by the fuel pump transmission shaft. NTS6ZLCz-129 dual-fuel turbocharged intercooled engine, in $1000 \mathrm{~h}$ durability test, fuel pump transmission shaft fractured suddenly. Fuel pump could not supply oil, the engine stopped. The fractured transmission shaft is shown in Fig. 1. The transmission shaft is made of $40 \mathrm{Cr}$, fracture diameter is $30 \mathrm{~mm}$, and manufacturing process consists of cutting, forging, roughing, quenching and finishing.

To identify shaft fracture causes, fracture analysis, chemical analysis, microstructure examination and finite element stress analysis were carried out on the fractured shaft.

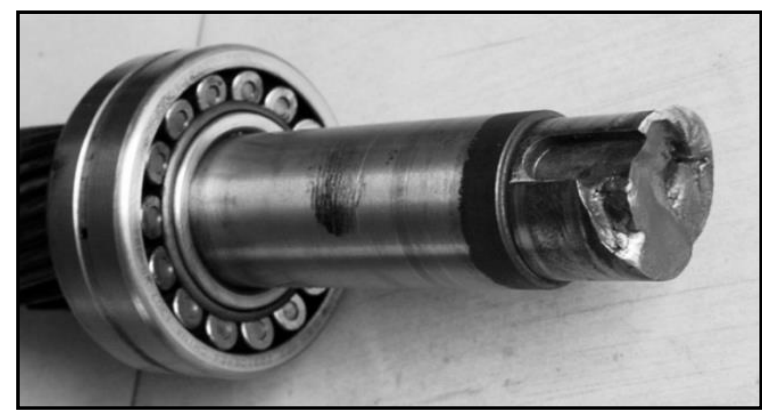

Figure 1. Fractured fuel pump transmission shaft

\section{Inspection and analysis}

\subsection{Fracture macroscopic analysis}

Fracture surface rust obviously, certain areas have bumps. Specimens were cut from fracture surface, fracture surface observed after ultrasonic cleaning with alcohol. Macro picture of fracture is shown in Fig. 2. The fracture surface was rough, around the bumps and final crack area had obvious plastic deformation, the other parts had no significant deformation. Fracture in most regions showed obvious macroscopic fatigue Bay Ridge. According to the shape of the distribution of fatigue Bay ridges, the path of crack extension, the crack source faults and final fracture zone can be determined. The source region of the crack and the final fracture zone are shown in Fig. 2. The crack source area is located in a small longitudinal cross section. This section is close to the keyway site and nearly vertical to step surface and the main fracture. Because of the crack source area had obvious impact on the abrasion, obvious macroscopic defects failed to be observed. The final fracture zone is located on the circumference, opposite to the source region of crack, and has a smaller shear lip. Since the fracture is not well protected, no obvious rapid tear streaks are observed in the final fracture zone except in the cut lip.

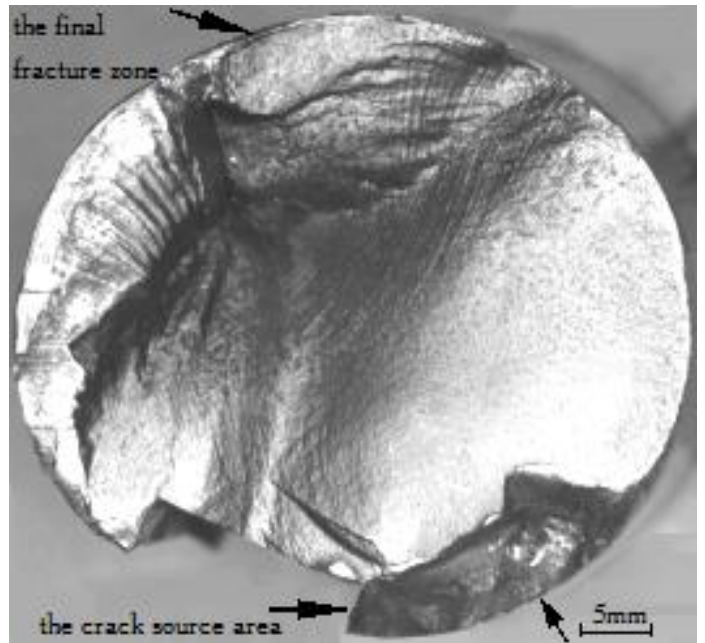

Figure 2. Macro picture of fracture

Take the area near the final fracture as the rapid tear streaks area before the final fracture. This area has no obvious macroscopic fatigue Bay ridges. This area is very small, and takes a small proportion of the total area of the 
fracture. From the above examination and fracture analysis, it can be determined that the fracture of the shaft is caused by low stress and high cycle fatigue in the keyway.

\subsection{Metallographic examination}

Three metallographic specimens are cut from the fracture surface area, which is vertical to the main fracture surface through the source region of the crack. Etchant is $4 \%$ Nital.

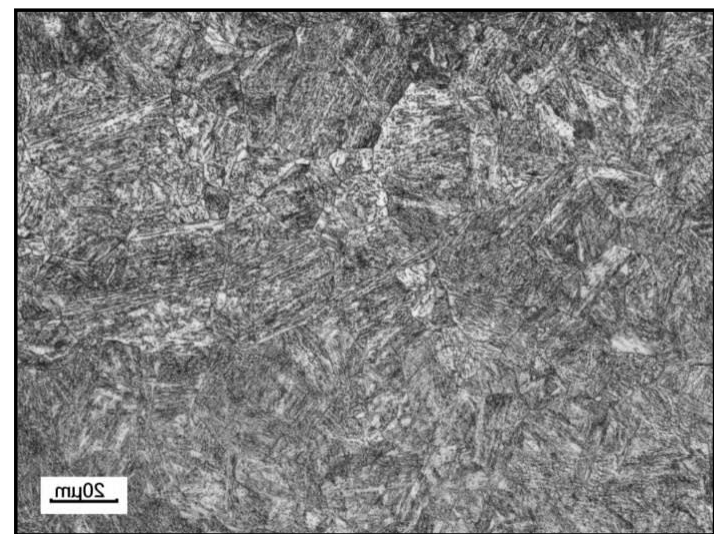

Figure 3. Microstructure organization of transmission shaft (Magnification of 500, 4\% Nital)

Fig. 3 illustrates the microstructure of fractured transmission shaft is tempered sorbite. Grain-size number is 8 according to GB / T 6394-2002, and it is the normal quenching and tempering heat treatment state organization.

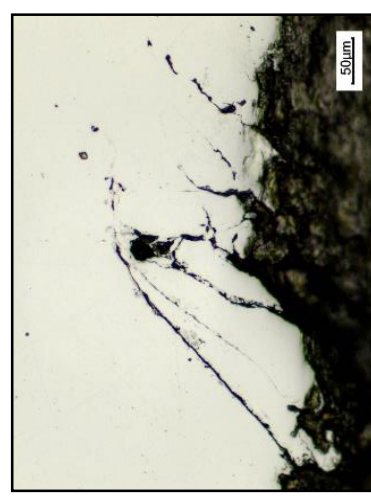

(a) Magnification of 200 No etchant

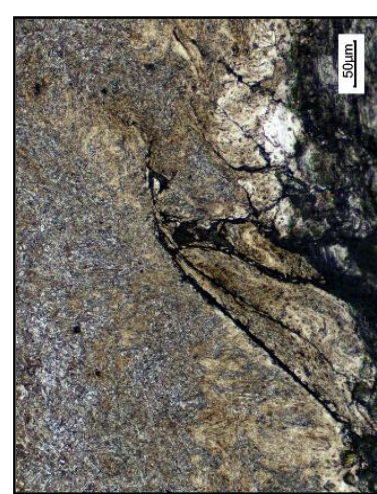

(b) Magnification of 200 $4 \%$ Nital
Figure 4. Surface defects and microstructure of the fracture surface in the source region of the crack

Fig. 4 illustrates there are cracked defects oblique stretched into internal in the crack source region. Oxide inclusion exists in the fracture surface, inner and surrounding areas of defects. Nearby the fracture surface and cracked defects, there is obvious decarbonization phenomenon. The defect has forging or rolling folding defect characteristics.

Fig. 5 illustrates there are cracked defects oblique and smooth stretched into internal. Oxide inclusion exists in the fracture surface and inner area of defects. There are continuous tiny dots and granular inclusions on both sides near the crack. The defect has forging or rolling folding defect characteristics.

Fig. 6 is a partially enlarged view of Fig. 5. Fig. 6 illustrates oxide inclusion exists in the fracture surface and inner area of defects. Continuous tiny dots and granular inclusions on both sides near the crack are clearly visible.

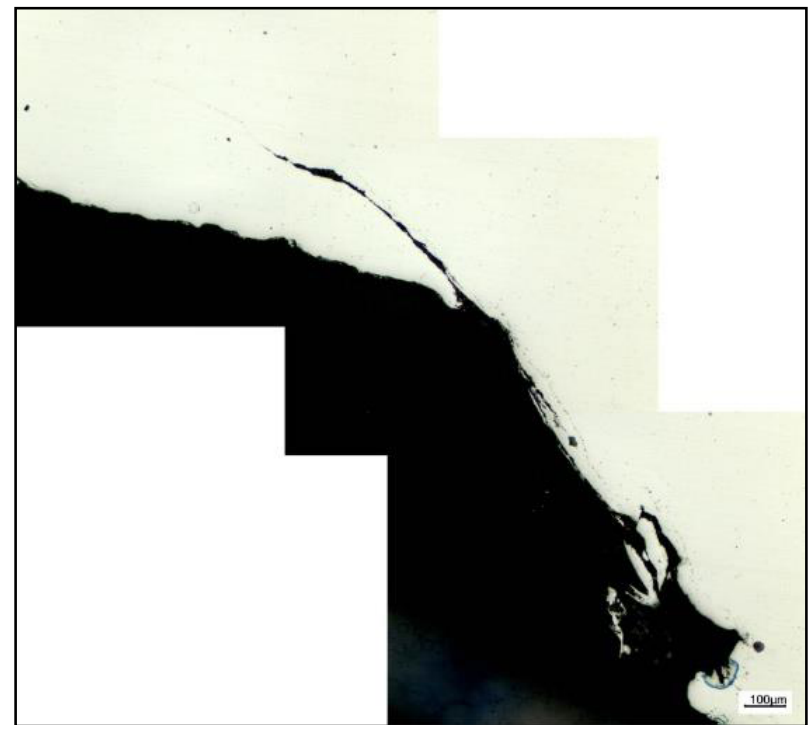

Figure 5. Surface defects of the fracture surface in the source region of the crack (Magnification of 500, no etchant)
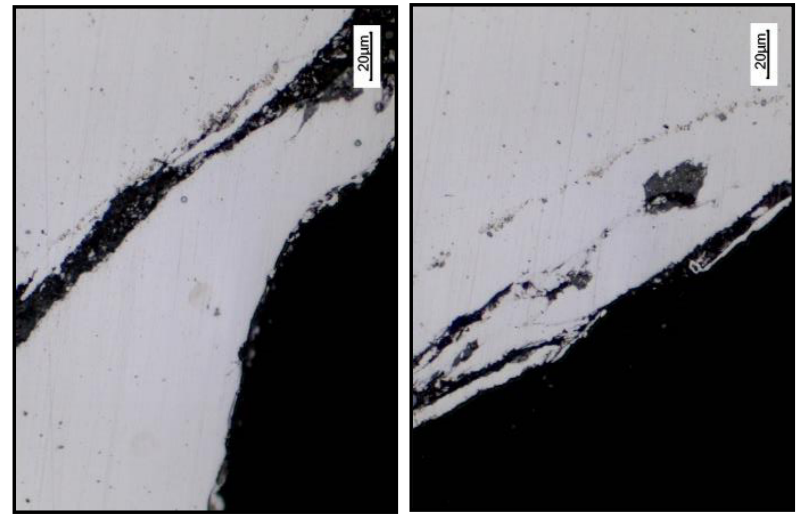

Figure 6. Surface defects of the fracture surface in the source region of the crack (Magnification of 500, no etchant)

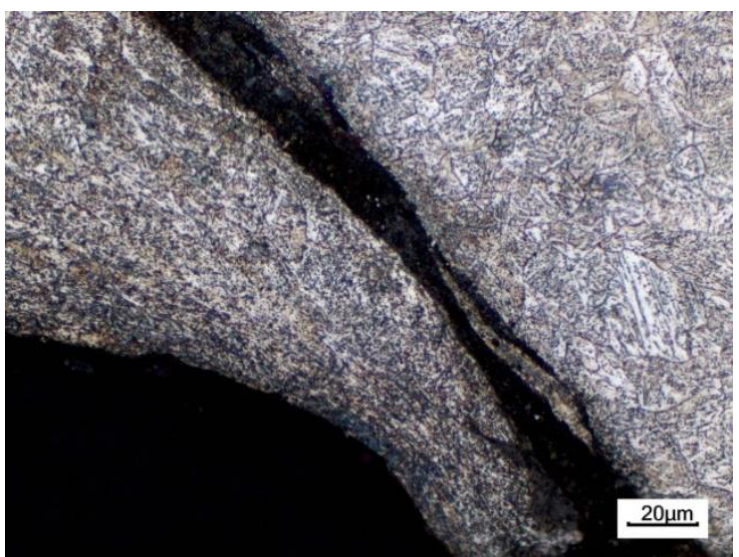

Figure 7. Surface defects and microstructure of the fracture surface in the source region of the crack (Magnification of 500, $4 \%$ Nital) 
The microstructure in Fig. 7 obtained after etching in $4 \%$ Nital. There are decarbonization in the surface and both sides of the crack.

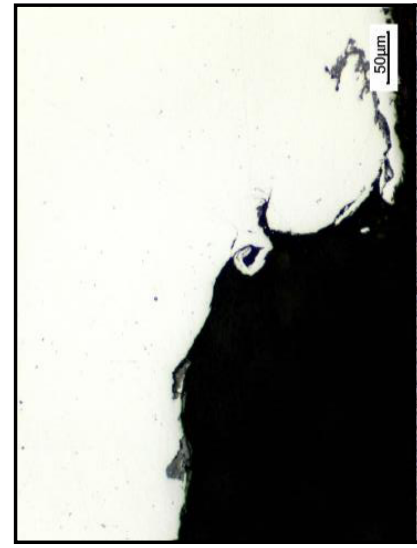

(a) Magnification of 200 No etchant (b) Magnification of 500 $4 \%$ Nital

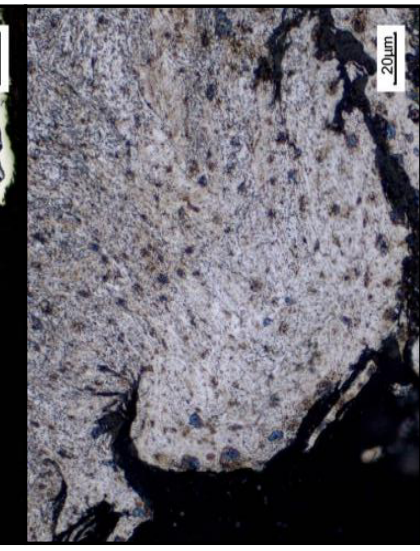

Figure 8. Surface defects and microstructure of the fracture surface in the source region of the crack

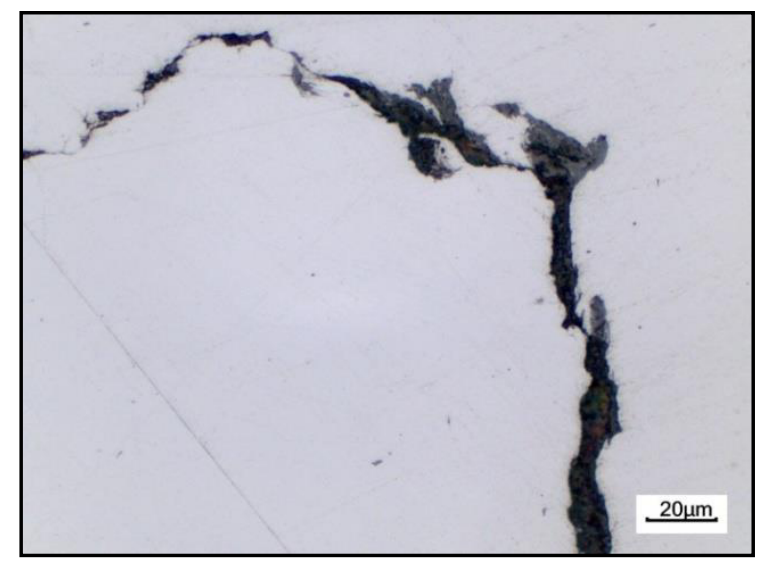

Figure 9. Internal cracks of the fracture surface nearby the source region of the crack (Magnification of 50, no etchant)

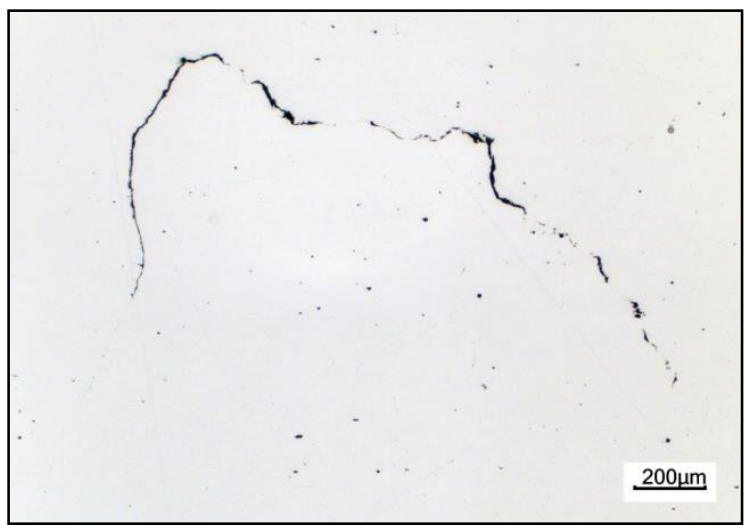

Figure 10. Internal cracks of the fracture surface nearby the source region of the crack (Magnification of 50, no etchant)

Fig. 8 illustrates there are curling defects and oxides in the crack source region. Obvious decarbonization exists in the surface organization, thus shows that this defect has forged or rolled folding defect characteristics.

Fig. 9 illustrates there are internal cracks nearby the source region of the crack. The crack snaked stretches and has a tapering tail. There are oxide inclusions inside the crack.
Fig. 10 is a partially enlarged view of Figure. Internal crack is filled with oxide, it can be seen clearly.

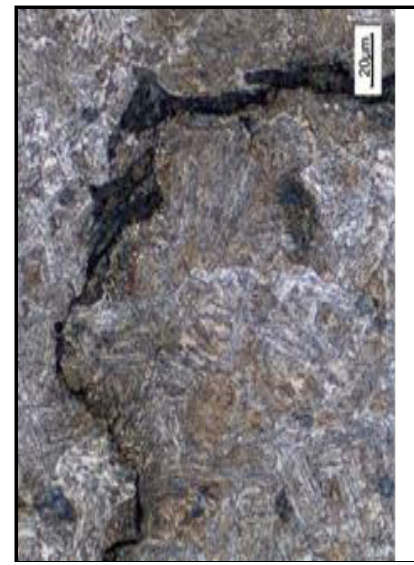

(a) fatigue crack

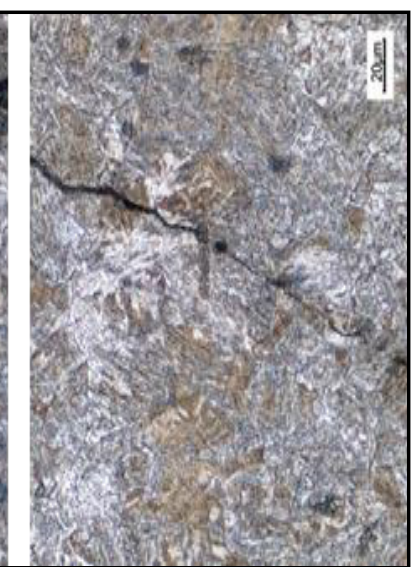

(b) fatigue crack tail
Figure 11. Internal cracks and microstructure of the fracture surface nearby the source region of the crack (Magnification of $500,4 \%$ Nital)

Fig. 11(a) is microstructure of fatigue crack, and Fig. 11 (b) is the microstructure of fatigue crack tail. Except the wider parts of the crack, the crack is mainly dominated by the extension of transgranular crack. This crack may be an extension of the original crack in heat treatment, and has a tapering tail. The crack should be fatigue extension during service of the transmission shaft.

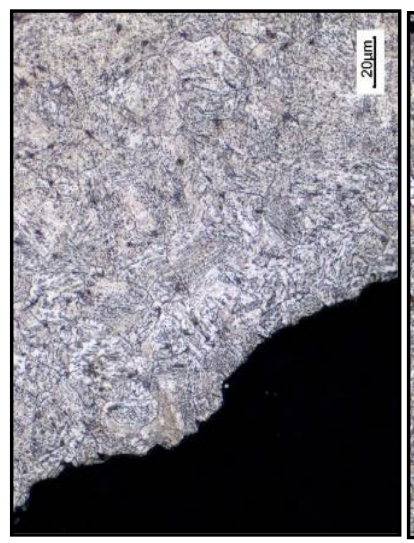

(a) fatigue crack

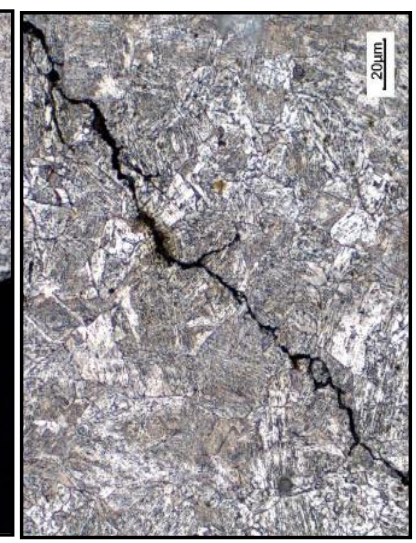

(b) fatigue crack tail
Figure 12. Fatigue Crack Growth Area and organization (Magnification of 500, 4\% Nital)

Fig. 12 (a) is microstructure of fatigue crack, and Fig. 12 (b) is the morphology and microstructure of fatigue crack tail. Fig. 12 illustrates the fatigue crack is mainly transgranular cracks. The microstructure is tempered sorbite, which belongs to the normal quenching and tempering heat treatment microstructure.

\subsection{Analysis of the chemical composition}

Specimens were cut from fracture surface, and SPECTRO MAXx optical direct-reading spectrometer was used to analysis chemical composition, as observed in Table 1. It was shown that chemical composition of the transmission shaft met the requirements of GB/T 3077 1999 for $40 \mathrm{Cr}$. 
Table 1. Chemical composition test results (\%)

\begin{tabular}{|c|c|c|}
\hline Item & Measured value & Technical specifications project \\
\hline $\mathrm{C}$ & 0.40 & $0.37 \sim 0.44$ \\
\hline $\mathrm{Si}$ & 0.24 & $0.17 \sim 0.37$ \\
\hline $\mathrm{Mn}$ & 0.60 & $0.50 \sim 0.80$ \\
\hline $\mathrm{Cr}$ & 0.99 & $0.80 \sim 1.10$ \\
\hline $\mathrm{P}$ & 0.009 & $\leq 0.035$ \\
\hline $\mathrm{S}$ & 0.008 & $\leq 0.035$ \\
\hline
\end{tabular}

\section{Finite element analysis}

The CREO 3D software of PTC Company can be used to test, study and improve the product design in the virtual condition. [1] In this study, CREO are used for strength check of fuel pump transmission shaft.

\subsection{Boundary conditions and loads}

With any FEA package, accurate loadestimation depends on the quality of the modelbuilt by the analyst. [2] Using CERO, transmission shaft $3 \mathrm{D}$ model containing axial keyway and shoulder were built as observed in Fig. 13. Canters of the two key-ways are used as the two fixed ends. Transfer torque is 380 N.m, the diameter is $30 \mathrm{~mm}$ and the material is $40 \mathrm{Cr}$.

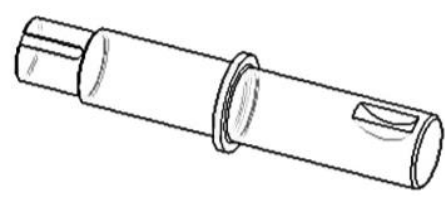

Figure 13. Three-dimensional model of transmission shaft

\subsection{Static analysis}

The static analysis is mainly used to analyze the maximum stress of the transmission shaft. [3] Fig. 14 shows the shear stress distribution and the maximum stress of transmission shaft. The maximum stress is $198.1 \mathrm{MPa}$ at the flat key, and the allowable shearing stress for $40 \mathrm{Cr}$ is $227 \mathrm{MPa}$. Obviously, the maximum stress is less than the allowable shearing stress. Thus the strength of the fuel pump transmission shaft meets the requirements.

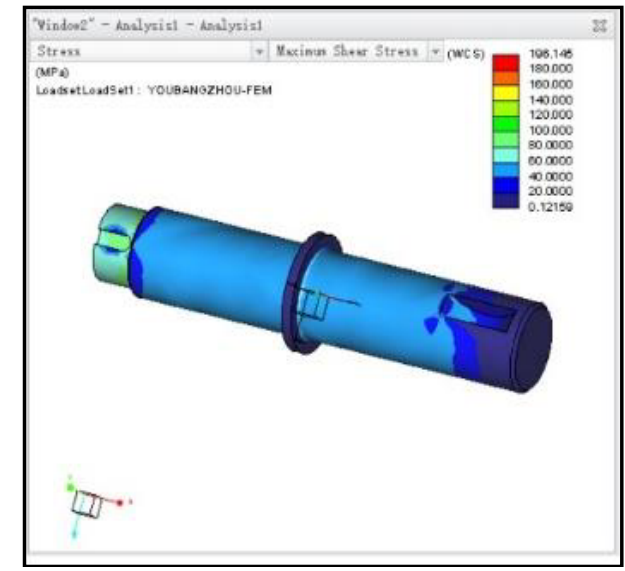

Figure 14. Shear stress distribution of transmission shaft

\section{Results and improvement}

Fracture analysis, chemical analysis, microstructure examination and finite element stress analysis were carried out on the fractured shaft. The analysis results show that the microstructure of transmission shaft is the normal quenching and tempering heat treatment state organization and the chemical composition of the shaft meets the requirements of GB/T 3077-1999 for 40Cr. In the crack source region, there is a tail round blunt crack type defect. This defect is at a certain angle with the external surface of parts and stretches into internal. Internal crack is filled with oxide inclusions. And there are obvious decarbonization oxidation and in the surface and both sides of the crack. This type defect is a typical forging or rolling folding defect. Shaft fracture is a type of low stress and high cycle fatigue fracture which is caused by a forging or folding defect on the outer circle near keyway. [4]The fracture mechanism is that there is a forging defect on the outer circle near keyway under long-term alternative working stress. The folding defect tip expands and leads to fatigue crack. With the increase of the number of stress, the crack continues to expand, when it expands to critical dimension, the actual bearing area is reduced. The remaining cross-sectional area cannot withstand the load, eventually led to the shaft fracture immediately.

The main causes of forging and folding of shaft parts are as follows:

(1) Due to the difference in the diameter of the metal at the step, the metal flow is poor. The forgings rotate during forging process, most metals flow along the tooling thus form a step, the rest of the metals are bent and pasted on the surface of forging because of the thin thickness. Thus they are pressed into the metal blank and formed folding in the subsequent forging forming process.

(2) The existence of the chamfer can reduce the stress concentration and make the flow smooth. However, the chamfer will produce a step gap and lead to the formation of folding easily.

(3) During the end stretching process, when the feed amount is very small and the press-fitting is large, the local deformation of the upper and lower ends of the metal is easy to form a folding.

According to the fuel pump shaft fracture position, it is preliminarily concluded that the shaft end stretching and small amount of feed lead to the folding defects. [5] Then, shaft forging process checked, and feed amount found indeed too small when stretching the shaft end. The pushing amount is too large, feed and unilateral compression ratio $L /(\triangle h / 2)$ is less than 1 , thus leads to a large metal deformation on surface layer and the end concave into a fold. Increase the amount of feed, when the feed unilateral compression ratio reached 1.2 , the transmission shaft was reprocessed. By ultrasonic inspection, no folding defects were found. The shaft was installed and the durability test was carried out, then the fuel pump transmission shaft worked properly.

\section{Conclusions}


The main conclusions can be drawn as follows:

(1) The finite element analysis shows that the strength of the fuel pump transmission shaft meets the requirements.

(2) Chemical analysis shows that the chemical composition of the shaft meets the requirements of GB/T 3077-1999 for 40Cr.

(3) Metallographic analysis shows that there are cracked defects oblique stretched into internal in the crack source region. Internal crack is filled with oxide inclusions. And there are obvious decarbonization oxidation and in the surface and both sides of the crack. This type defect is a typical forging or rolling folding defect.

(4) Forging process analysis shows that inappropriate parameters of forging process lead to folded forging defects on the outer circle near Keyway. Thus the fuel pump transmission shaft occurs low stress and high cycle fatigue fracture. By changing forging parameters, the problem of fractured shaft was effectively solved.

\section{Acknowledgment}

This work is supported by Nantong Shipping College school level key project "Fishing LNG / diesel dual fuel engine NOX Emissions Research" (project number: HYKJ2015 / A02).

\section{References}

1. H.R. Sheng, Jixie Gongchengshi/Mechanical Engineers 2, 144 (2015)

2. C.S. Baviskar, R. M. Tayade, G. Vinay, Int. J. Mech. Eng. \& Rob. Res 1(3), 22 (2012)

3. M. Shafaee, E. Abbas, F. Hamed, B. Jebraiel, Int. J. Mech. Eng. \& Rob. Res 5(2), 133 (2016)

4. Q.R. Xiang, Keji xiangdao/Technology Guide 2, 51 (2011)

5. J.C. Huang, Qiye Jishu Kaifa /Enterprise Technology Development 32, 104 (2014) 